\title{
When are semantic targets detected faster than visual or acoustic ones?*
}

\author{
FREDERICK BALL, CHRISTINE WOOD, and EDWARD E. SMITH \\ Stanford University, Stanford, California 94305
}

\begin{abstract}
Three experiments explored G. Cohen's (1970) finding that semantic targets can be detected faster than visual or acoustic ones when searching through meaningful prose. In all three experiments, the subject searched a sentence for a target defined visually, acoustically, or semantically. In Experiment I, the subject detected semantic targets faster than visual- or acoustic-syllable targets, regardless of whether he was instructed to read the sentence for meaning or simply scan it. Experiments II and III showed that this advantage of semantic targets over visual or acoustic ones was greatly reduced when all three types of targets were words. The results were interpreted as indicating that when words are presented in context, either: (a) the unit of word perception is the entire word, or (b) the unit of identification or awareness is at least as large as the entire word.
\end{abstract}

If a word is in our lexicon, we can usually recognize it in print, pronounce it, and offer something of a definition of it. That is, the psychological representation of a familiar word contains information about its visual, acoustic-articulatory, and semantic aspects (or codes). Given this, the question arises as to whether there are differences in the order in which these three types of codes are accessed when one reads a word. Since the word is visually presented, logic dictates that the lexicon must be accessed by visual information. Consequently, we would expect the visual code to be the first one available, while the access ordering of the acoustic and semantic codes remains an open question.

Recently, Cohen has performed a pair of experiments which bear directly on these issues and which, when taken together, yield a puzzling outcome. In her first experiment, Cohen (1968) presented a subject with a pair of one-syllable words and required him to decide whether the pair were identical with respect to one of three criteria-visual, acoustic, or semantic (for the semantic criterion, identity meant synonymy). Cohen found that the subject's decisions were fastest for the visual criterion and slowest for the semantic one. Thus the visual code was apparently accessed first, the semantic code last. Cohen's (1970) second experiment dealt, in part, with the same issue, but now the task involved reading through meaningful material. In this study, a subject was first given a target classified as visual (e.g., the letter d), acoustic (e.g., the sound corresponding to $/ d /$ ), or semantic (e.g., any instance of the animal category). Then the subject searched for the specified target while reading a series of sentences for meaning;

*This research was supported in part by United States Public Health Service Grant 19705. Requests for reprints should be sent to Edward E. Smith, Dispartment of Psychology, Stanford University, Stanford, California 94305. when a target item (e.g., dog) was encountered, the subject was directed to cancel it out. In contrast to the previous results, semantic targets were now detected faster than visual ones, which in turn were detected more quickly than acoustic targets. There are two surprising aspects of these latter results. First, embedding the targets in context produced an access order for the three codes which differed from that obtained in the absence of context (Cohen's earlier study). Second, contrary to logical considerations, semantic targets were found faster than visual ones (as well as acoustic ones). This latter finding will be referred to as the semantic-superiority effect, and one purpose of the present paper is to explicate this effect and its dependence on context and other factors. ${ }^{1}$

\section{Determinants of the Semantic-Superiority Effect}

In our attempt to understand the semanticsuperiority effect, we first made the obvious assumption that this effect tells us something about the initial visual coding of a word. Obvious though it may be, we shall later have cause to question the assumption in the Discussion section. In any event, given this assumption, we then focused on two perceptual explanations of the semantic-superiority effect that had been alluded to by Cohen (1970) in her context experiment. The first explanation was concerned with variations in the functional units of word perception, and the second dealt with selective effects of semantic context on word perception. These are considered in turn.

Our first hypothesis was that the semanticsuperiority effect was really due to a variation in the size of perceptual units. This requires some explanation. Consider Cohen's (1970) context experiment and recall that here the visual and acoustic targets were defined by single letters and phonemes, respectively. These targets may well have been smaller in size than the perceptual units the subject 
typically employs in perceiving words, for recent findings on the visual and auditory perception of isolated words indicate that syllables, rather than letters or phonemes, may be the critical units of perception (Massaro, 1972; Spoehr \& Smith, 1973). If syllables also function as perceptual units for words in context, then in Cohen's task a subject given either a letter or phoneme target would have first had to process each word in the test sentences on the basis of its syllables, and then had to "back up" or decompose each syllable into its constituent letters or phonemes. Such a retrograde analysis would have been needed for each word encountered, and this might have required appreciable time. For the semantic targets in this experiment, the target (a word instancing a semantic category) was at a higher level than a syllable, consequently necessitating a progressive synthesis from the syllable level. But this progressive synthesis might have required less time than the retrograde analysis needed with the other types of targets, since the former process is a well-practiced one frequently used in reading. Granting all of these assumptions, a semantic-superiority effect is to be expected in Cohen's context study. In contrast, in Cohen's (1968) earlier study of isolated words, the items were all one-syllable words regardless of whether a visual, acoustic, or semantic decision was called for. Hence, there was no need for a retrograde analysis of visual and acoustic items, and consequently the absence of a semantic-superiority effect here is understandable.

The above perceptual units hypothesis is completely post hoc, but it does lead directly to a testable prediction for a context task. If the visual and acoustic targets are defined in terms of syllables, then these targets would no longer necessitate a retrograde perceptual analysis and the semantic-superiority effect should disappear. This prediction was tested in Experiment I.

Our second hypothesis about the semanticsuperiority effect focused on the influence of meaningful context on word perception. Again, we are chiefly concerned with Cohen's context study, but this time we will suggest that the semantic-superiority effect was due to a facilitation in the semantic target condition rather than to a retardation in the visual and acoustic target conditions. In the semantic condition, the definition of a target is compatible with the auxiliary reading task, as both are semantic in nature, and consequently the subject may have been induced to use the semantic information obtained from reading the context to help him find the targets. Since relevant semantic information can facilitate word perception (Morton, 1969; Tulving \& Gold, 1963), semantic targets should be detected relatively quickly. When the targets were visual or acoustic, however, their definitions were not compatible with the basically semantic nature of the reading task, and thus the subject may not have used the semantic information obtained from context to facilitate word perception. Hence, visual and acoustic targets should have been detected relatively slowly, and this would give rise to the semantic-superiority effect. The results of Cohen's (1968) earlier study are in accord with this interpretation since there was no meaningful context which could selectively facilitate detection of semantic targets.

This semantic facilitation hypothesis thus offers another post hoc explanation of the relevant data. In this case, the hypothesis may be tested experimentally by assessing the assumption that reading the search material for meaning facilitates the simultaneous search for semantic targets. If, in a context task, a subject can be prevented from reading for meaning, then the semantic-superiority effect should disappear. This prediction was tested in Experiment I as follows. The material to be searched consisted of either a meaningful sentence or an ungrammatical and nonsensical string of words, and the subject was instructed either to read the material for meaning while scanning it or simply to scan it. According to the semantic facilitation hypothesis, the semanticsuperiority effect should obtain only when the material consists of a sentence and the subject is instructed to read it for meaning.

\section{EXPERIMENT I}

\section{Method}

Overview. On each trial, the subject was presented with an 11-word string and had to search through it for a target defined either visually, acoustically, or semantically. One group of Ss was instructed to read each string for meaning while scanning for the target (the Read group), and a second group was told to ignore any semantic content and to simply scan for the target (the Scan group). For each of these groups, half of the strings presented were sentences while the rest consisted of scrambled versions of these sentences (i.e., nonsentences). For both sentences and nonsentences, the target was a visual syllable, an acoustic syllable, or a semantic category. Thus the basic design was a mixed factorial, with instructions (Read vs Scan) as a between-subject factor, and type of material (sentence vs nonsentence), type of target (visual vs acoustic vs semantic), and position of target within the sentence as within-subject factors.

The relevant predictions from the two hypotheses of interest can be summarized easily. According to the perceptual units hypothesis, there should not be any semantic-superiority effect, and this prediction is independent of the nature of the instructions or the type of material. The rationale is simply that the use of syllables as visual and acoustic targets should be sufficient to eliminate any advantage of the semantic targets. For the semantic facilitation hypothesis, the predictions depend on the instructions and material type. There should not be any semantic-superiority effect for the Scan group (regardless of whether they are scanning sentences or nonsentences) because these Ss are not reading for meaning. But, by the same rationale, the Read group should show a semantic-superiority effect when presented with sentences, though not when given nonsentences. Thus the predictions from the two hypotheses overlap considerably and emphasize a negative finding, i.e., the absence of a semantic-superiority effect. Although this experimental approach is not completely desirable. the results were. so unambiguous that no problems of interpretation arose.

Subjects. The subjects were 36 Stanford Undergraduates (22 males and 14 temales) who participated in the experiment to fulfill 
a course requirement. Half the subjects were randomly assigned to the Read group and the rest were assigned to the Scan group.

Materials. Fifty-four two-syllable concrete nouns were selected from Kučera and Francis (1967) to serve as critical words. (By a critical word, we mean either the word instancing a semantic target or the word containing a visual or acoustic syllable target.) These words were then randomly divided into nine sets of six each, where the items in a particular set were designated as critical words to appear in one of the nine internal positions of an 11-word sentence. Next, 54 11-word sentences were generated, 6 each with a critical word in Position i, where i varied from 2 to 10 . All sentences were simple declaratives, and each had the form agent-action-object, e.g., The game warden followed the migratory salmon for the whole year. Finally, 54 11-word nonsentences were created by randomizing the position of all of the words in a sentence, except the critical word, so that the resulting string was ungrammatical and nonsensical. Thus the serial position of a critical word in a nonsentence was the same as its position in the comparable sentence.

Consider now the six specific sentences associated with each of nine serial positions. Each of these sentences was used with each of the three target types. Thus one subject received a particular sentence associated with a visual target, another received the same sentence with an acoustic target, and a third received the same sentence with a semantic target. For the corresponding nonsentence, three new subjects were needed, and these six groups of subjects formed a Latin square with the factorial combination of target type and material type (sentence or nonsentence).

For a particular critical word (e.g., salmon), the semantic definition of a target consisted of the name of its superordinate category (fish) as determined by the Battig and Montague (1969) norms. For this same critical word, the visual target consisted of the letters comprising either the first or second syllable of this word; first syllables were used as targets for half of the critical words, and second syllables for the rest. Finally, for this same critical word, the acoustic target corresponded to the pronunciation of the visual target. To insure that $\mathrm{S}$ did not convert an acoustic target into its visual equivalent, approximately $40 \%$ of the acoustic targets were chosen so that their spelling could not be predicted from their pronunciation. For example, the spelling of the first syllable of salmon cannot be predicted from the acoustic description /saem/. Also, the subject was explicitly cautioned that translating an acoustic target into a visual one might mislead him.

Procedure. Each subject was tested individually. At the beginning of a session, the subject read through a series of instructions which contained a description of the task and materials. The procedure was then further explained with a step-by-step demonstration, followed by 11 practice trials.

Consider first the procedure for the Read group. At the start of each trial, the experimenter verbally specified the target for that trial, e.g., the letters $S, A, L$, and $M$, or the pronunciation $/ \mathrm{saem} /$, or an instance of the category fish. The subject then focused on a fixation point in a Gerbrand's two-channel tachistoscope. When he was ready, he pushed a start button held in his left hand. One-half second later, the test string (sentence or nonsentence) for that trial was displayed, with the beginning of each sentence placed just to the right of the fixation point. A Standard timer was initiated concurrently with the presentation of a string. Each test string was typed in two lines on a $6 \times 9$ in. blank index card, centered for tachistoscopic display. The subject then read the string for meaning, in a left-to-right and top-to-bottom manner, until he encountered a target, at which point he depressed a response button that terminated the display and stopped the timer. Every string contained exactly one target. Following his response, the subject verbalized the critical word; in addition, the subject then answered a simple true-false question about the content of that test string. The purpose of this question was to encourage the subject to read sentences and nonsentences for meaning even if he frequently did not read much of the string before finding the target.

The procedure for the Scan group was identical to the above, with two exceptions. First, rather than being instructed to read each string for meaning, a subject in the Scan group was told to scan each string for the target in a left-to-right and top-to-bottom manner. In fact, he was explicitly directed to ignore the content of each string as he "might find it distracting." Second, a subject in this group was never asked a true-false question about the content of the string.

\section{Results}

Table 1 presents mean correct RTs to find the target as a function of the three main factors of interest-instructions (Read or Scan), material type (sentences or nonsentences), and target type (visual, acoustic, or semantic). The reader will recall that the perceptual units hypothesis predicts no semanticsuperiority effects in any condition, while the semantic facilitation hypothesis predicts that only the combination of Read instructions and sentence materials should lead to a semantic-superiority effect. It is abundantly clear from Table 1 , however, that all four combinations of instructions and material type result in a semantic-superiority effect. That is, semantic targets are detected faster than either visual syllable or acoustic syllable ones, regardless of whether the instructions required reading or scanning, or whether the material consisted of sentences or nonsentences. Thus both hypotheses are strikingly disconfirmed.

To analyze the results statistically, we used an analysis of variance for a one-between- and three-within-subject design, and computed min $\mathrm{F}$ ratios (symbolized, min $F^{\prime}$ ), as recommended by Clark (1973) for cases where both subjects and material are treated as random effects. The analysis indicated that all four main effects were significant. First, all search RTs increased monotonically with the serial position of the target in the string, $\min F^{\prime}(8,114)=54.96, p<.001$. This result simply indicates that $S$ s followed our instructions to search the test string in a left-to-right and top-to-bottom manner, and to terminate his search as soon as he found the target. Second, the subjects detected targets more rapidly when instructed to scan $(1.43 \mathrm{sec})$ than when instructed to read $(1.64 \mathrm{sec}), \min F^{\prime}(1,1064)=$ $6.84, \mathrm{p}<.001$. This finding again attests to the fact that $\mathrm{S}$ complied with our instructions, as the Read

Table 1

Mean, Correct RTs (in Seconds) as a Function of Instructions, Material Type, and Target Type (Experiment I)

\begin{tabular}{llcccc}
\hline \multirow{2}{*}{$\begin{array}{l}\text { Instruc- } \\
\text { tions }\end{array}$} & $\begin{array}{c}\text { Material } \\
\text { Type }\end{array}$ & \multicolumn{4}{c}{ Target } \\
\cline { 2 - 6 } & Visual & Acoustic & Semantic & Mean \\
\hline \multirow{3}{*}{ Read } & Sentence & 1.61 & 1.61 & 1.42 & 1.55 \\
& Nonsentence & 1.74 & 1.78 & 1.66 & 1.73 \\
& Mean & 1.68 & 1.70 & 1.54 & 1.64 \\
& Sentence & 1.45 & 1.47 & 1.26 & 1.40 \\
Scan & Nonsentence & 1.46 & 1.60 & 1.33 & 1.46 \\
& Mean & 1.46 & 1.54 & 1.30 & 1.43 \\
\hline
\end{tabular}


group was required to perform two tasks (comprehension and target detection) and thus should have taken longer than the Scan group, who had to perform only one task (target detection). Third, the subjects detected targets more rapidly when searching through sentences $(1.47 \mathrm{sec})$ than nonsentences $(1.59 \mathrm{sec}), \min \mathrm{F}^{\prime}(1,914)=22.54, \mathrm{p}<.001$. This result was qualified by an interaction with instructions which will be discussed below. Fourth, Ss found semantic targets $(1.42 \mathrm{sec})$ faster than visual $(1.57 \mathrm{sec})$ or acoustic ones $(1.62 \mathrm{sec})$, min $\mathrm{F}^{\prime}(2,414)$ $=17.38, \mathrm{p}<.001$. This last main effect was elucidated by selected contrasts which showed that though semantic targets were detected significantly faster than visual or acoustic ones $\left[\min \mathrm{F}^{\prime}(1,394) \doteq\right.$ $24.23, \mathrm{p}<.001]$, visual and acoustic targets did not differ significantly in their detectability [min $\mathrm{F}^{\prime}$ $(1,393)=2.02, \mathrm{p}>.10]$. This semantic-superiority effect is sufficient to discredit the perceptual units hypothesis.

The critical predictions from the semantic facilitation hypothesis are all reflected in interactions between target type, on the one hand, and instructions, material type, or their interaction, on the other. All three such interactions were clearly insignificant, $\min \mathrm{F}^{\prime}<1$ in each case, which provides statistical documentation for the fact that the semantic-superiority effect occurred in all possible cases (see Table 1). In fact, the only significant interaction was that between instructions and material type, $\min \mathrm{F}^{\prime}(1,473)=5.07, \mathrm{p}<.025$. This interaction was such that the superiority of sentences over nonsentences was less in the Scan group than in the Read group (see Table 1). Selected contrasts showed that the interaction was due to the fact that subjects in the Read group processed sentences significantly faster than nonsentences, 1.55 vs $1.73 \mathrm{sec}$, respectively $\left[\min \mathrm{F}^{\prime}(1,474)=25.10\right.$, $p<.001]$, while $S$ s in the Scan group processed sentences and nonsentences at comparable rates, 1.40 vs $1.46 \mathrm{sec}$, respectively $\left[\min \mathrm{F}^{\prime}(1,474)=3.35\right.$, $.05<\mathrm{p}<.10$ ]. These findings again indicate that our instructional variation was successful. That is, we would expect that scrambling the order of a sentence would have more of an effect on reading than scanning, since the subject had to determine meaning only in the former task.

Finally, some mention should be made of error rates. A subject was assumed to be in error when he thought there was no target or when he depressed that response button but then reported a word other than the critical one (i.e., thought the wrong word was the target). Combined, these errors totaled only $6.4 \%$ of all trials. The error rate was somewhat lower for subjects in the Read group (5.4\%) than for those in the Scan group $(7.2 \%)$. Within the Read group, the subjects made fewer errors on sentences $(3.9 \%)$ than on nonsentences $(6.8 \%)$, and fewer errors on semantic and visual targets $(4.6 \%$ and $4.3 \%$, respectively) than on acoustic ones $(7.1 \%)$. In the Scan group, the subjects also made fewer errors on sentences $(6.8 \%)$ than on nonsentences $(8.0 \%$ ) and fewer errors on semantic and visual targets $(7.4 \%$ and $6.4 \%$, respectively) than on acoustic ones $(8.6 \%)$. Subsequent statistical tests indicated that the error rates for semantic targets were never significantly higher than the comparable rates for visual targets ( $\chi^{2}<1$, df $=1$, in all four cases). Thus there is no indication that the semantic-superiority effect can be attributed to a speed-accuracy tradeoff.

\section{Discussion}

The above experiment seems to have provided a fair test of both the perceptual units and semantic facilitation hypotheses, and both were disconfirmed by the occurrence of the semantic-superiority effect in all conditions. However, it is possible to salvage the perceptual units hypothesis by changing just one of its assumptions. Juppose that, when words appear in any kind of context, the critical unit of word perception is the whole word itself. Then our previous analyses of the semantic-superiority effect in earlier studies (pp. 1-2) would still stand, and the results of Experiment $I$ would also be explicable. In the latter experiment, the visual and acoustic syllable targets were still smaller than words, and consequently would have led to some time-consuming retrograde analysis. This revised version of the perceptual units hypothesis can be tested by altering the task used in Experiment I so that targets defined visually or acoustically are whole words rather than syllabic components of words. In this case, search for the visual and acoustic targets should no longer require any retrograde analyses, and the semantic-superiority effect should be eliminted. This prediction was tested in Experiment II.

\section{EXPERIMENT I}

\section{Method}

Overview. The basic task was similar to the Read-sentence combination of Experiment I. On each trial, the subject was presented with a nine-word sentence and told to attempt both to read it for meaning and to scan it for a target. Again, the target was a visual syllable, an acoustic syllable, or an instance of a semantic category. The major variation in this study concerned the relation between the visual and acoustic targets and their corresponding critical words (i.e., the words in the test sentence that contained the targets). On half the trials, the situation was the same as in Experiment 1 , as each critical word consisted of two syllables (where neither alone formed a word) and every visual and acoustic target was a one-syllable nonword. These trials will hereafter be referred to as nonword-search trials. On the rest of the trials, every critical word contained only one syllable and each visual and acoustic target was a one-syllable word that perfectly matched its corresponding critical word (hereafter, word-search trials). To illustrate, for the nonword-search trials, a critical word might be salmon, with the visual target being the letters $S, A, L$, and $M$, the acoustic target being the pronunciation $/ \mathrm{saem} /$, and the semantic 
target being an instance of the category fish. For the word-search trials, a critical word might be pear, with the visual target consisting of the letters P, E, A, and R, the acoustic target being $/ \mathrm{pe}^{\partial} \mathrm{r} /$, and the semantic target being an instance of the category fruit. Thus, this word- vs nonword-search variation involved altering both the content of the visual and acoustic targets (words or nonwords) as well as the number of syllables in the critical words (one or two syllables).

The predictions from our revised perceptual units hypothesis are as follows. For the nonword-search trials, there should be a semantic superiority effect, i.e., the results of Experiment I should be replicated. However, for the word-search trials, this effect should not occur. These predictions rest only on the assumpion that the effect of interest is due to the use of targets smaller than a word.

Subjects. The subjects were 27 Stanford undergraduates (17 males and 10 females) who participated to fulfill a course requirement. None had participated in Experiment $\mathrm{I}$.

Materials. Twenty-four two-syllable and 24 one-syllable concrete nouns were selected from Kučera and Francis (1967) to serve as the critical words for the nonword-and word-search trials, respectively. For the two-syllable words, neither syllable formed a word. Within each type, the 24 words were then randomly divided into four sets of 6 each; the words in a particular set were designated as critical words to appear in one of the four even-numbered positions of a 9-word sentence. A 9-word simple declarative sentence was then created for each critical word, resulting in 48 sentences which were balanced with respect to type of critical word (one or two syllables) and serial position. Again, every sentence was used with a visual, acoustic, and semantic target, but this time type of target was a between-subject variable. That is, every subject was presented the same 48 sentences, with nine subjects searching for visual targets, nine for acoustic targets, and the final nine for semantic targets.

Procedure. The procedure was the same as that used for the Read group in Experiment I with the following exceptions: (a) each sentence was typed on a single line on a $6 \times 9$ in. card, rather than on two lines; (b) target type remained constant, rather than being varied within-subject; and (c) half the trials involved word searches (rather than all trials requiring nonword search), and word-search and nonword-search trials were randomly intermixed.

\section{Results}

Table 2 presents mean correct RTs for each type of target, as a function of whether word or nonword search was required. The revised perceptual units hypothesis predicts that the semantic-superiority effect should be obtained with nonword search, but should be elimited or reduced when word search is involved. Table 2 offers some support for these predictions. When one considers only the contrast between semantic and visual targets, the superiority of the semantic case is less with word than with nonword search. For the contrast between semantic and acoustic targets, there is a substantial semanticsuperiority effect for the nonword search but much less of an effect for the word search.

An analysis of variance was used to provide statistical support for the effects observed in Table 2. Since this analysis was somewhat simpler than that employed in Experiment I, we used quasi-F ratios (symbolized $\mathrm{F}^{\prime}$ ), as recommended by Winer (1971). The analysis indicated that all three main effects were significant. First, the subjects detected targets faster the earlier the critical word occurred in the sentence, $F^{\prime}(3,88)=41.58, p<.001$. Again, this finding simply indicates that the subjects followed our
Table 2

Mean, Correct RTs (in Seconds) as a Function of Target Type and Type of Search (Experiment 11)

\begin{tabular}{lcccc}
\hline & \multicolumn{4}{c}{ Target Type } \\
\cline { 2 - 5 } & Visual & Acoustic & Semantic & Mean \\
\hline Word Search & 1.41 & 1.28 & 1.19 & 1.29 \\
Nonword Search & 1.65 & 1.50 & 1.18 & 1.44 \\
Mean & 1.53 & 1.39 & 1.18 & 1.36 \\
\hline
\end{tabular}

instruction to read and scan from left to right. Next, overall, target type influenced $\mathrm{RT}, \mathrm{F}^{\prime}(2,25)=3.86$, $p<.05$. Subsequent contrasts indicated that semantic targets were detected faster than visual or acoustic ones [1.18 vs 1.53 and $1.39 \mathrm{sec}$, respectively, $\left.F^{\prime}(1,25)=6.46, p<.05\right]$, but the latter two types of targets did not differ significantly $\left[\mathrm{F}^{\prime}(1,25)=2.68\right.$, $\mathrm{p}>.05]$. Third, the subjects detected targets faster during word search $(1.29 \mathrm{sec})$ than during nonword search $(1.44 \mathrm{sec}), \mathrm{F}^{\prime}(3,88)=9.90, \mathrm{p}<.01$.

The critical prediction of the hypothesis of interest is reflected in an interaction-that between the type of target and the type of search (word vs nonword). This interaction, readily apparent in Table 2, was significant, $F^{\prime}(2,36)=8.69, p<.01$. A NewmanKeuls test applied to the six means represented in Table 2 offered detailed evidence concerning our predictions. For nonword search, the subjects detected semantic targets $(1.18 \mathrm{sec})$ faster than either visual $(1.65 \mathrm{sec})$ or acoustic $(1.50 \mathrm{sec})$ ones, $\mathrm{p}<.01$ in both cases. In contrast, for word search there was no reliable difference between the semantic target and the acoustic one (1.19 and $1.28 \mathrm{sec}$, respectively, $p>.10$ ), and the difference between the visual target and its semantic counterpart (1.41 and $1.19 \mathrm{sec}$, respectively) was reduced, though still significant, $\mathrm{p}<.01$.

The overall error rate was only $2.2 \%$, and there was no indication that a speed-accuracy tradeoff could account for any of the main results. In view of the small number of errors; no attempt was made at a further analysis.

\section{Discussion}

While the data lend some support to our revised perceptual units hypothesis, there is one particularly anomalous finding-even on the word-search trials, the visual targets are still significantly slower than the semantic ones. A possible explanation of this finding is that on some of the word-search trials, the subjects may have treated the visually defined targets as a set of letters rather than as a word. That is, presenting a subject with the visual targets in a letter-by-letter manner (e.g., "The target consists of the letters P, E, $A$, and R") may have fostered a letter-search strategy. (See Smith \& Spoehr, 1974, for evidence on such strategies in word perception.) The assumption that subjects may have adopted such a latter strategy is made 
more plausible by the fact that subjects searching for visual targets never searched for acoustic or semantic ones. The latter two types of targets were clearly presented as words (e.g., the pronunciation / $\mathrm{pe}^{\mathrm{d}} \mathrm{r} /$ or an instance of the category fruit), and exposure to these target types might have eliminated a letter strategy in searching for visual targets. In light of these arguments, it follows that for word search, the superiority of semantic over visual targets should be eliminated if target type is varied within-subject. This prediction was tested in Experiment III.

Another purpose of Experiment III was to determine what would happen if the nonword-search trials for visual and acoustic targets were replaced by trials in which the target formed a one-syllable word (e.g., vent), but its corresponding critical word contained the target only as a part (e.g., convent). In this case, the subject's target is a word but the to-be-perceived critical word must be decomposed in order to match the target. Thus a retrograde analysis is needed, and our revised perceptual units hypothesis would seemingly predict that a semantic-superiority effect should occur here just as it does in nonword search.

\section{EXPERIMENT III}

\section{Method}

Overview. This experiment was identical to Experiment II, with two exceptions. First, target type was varied within-subject rather than between-subject. Second, the nonword-search trials were replaced by trials on which the subject searched for a one-syllable target (e.g., vent) included in a two-syllable critical word (e.g., convent), where the meaning of the latter was unrelated to the meaning of the target. We shall refer to these trials as the mixed-search trials.

Subjects. The 18 subjects ( 10 males and 8 females) were selected from the same population described in the earlier experiments. None of these subjects had particpated in the two previous studies.

Materials. Again, 48 sentences were used, 24 containing one-syllable critical words (used on the word-search trials) and 24 containing two-syllable critical words (used on the mixed-search trials). The former 24 were the same as those used in Experiment II. The 24 sentences containing two-syllable critical words were generated by the procedure used in previous experiments, except that each critical word contained a four-letter syllable which formed a word that was unrelated in meaning to the critical word. These four-letter syllables always served as targets for the mixed-search trials. The assignment of the two types of critical words to various serial positions in the test sentences was the same as in Experiment II. To insure that every sentence was used with every target type, we used a Latin-square design with three groups

Table 3

Mean, Correct RTs (in Seconds) as a Function of Target Type, and Type of Search (Experiment III)

\begin{tabular}{lcccc} 
& \multicolumn{4}{c}{ Target Type } \\
\cline { 2 - 5 } & Visual & Acoustic Semantic & Mean \\
\hline Word Search & 1.20 & 1.22 & 1.20 & 1.21 \\
Mixed Search & 1.24 & 1.28 & 1.20 & 1.24 \\
Mean & 1.22 & 1.25 & 1.20 & 1.22 \\
\hline
\end{tabular}

of subjects and the three target types comprising the dimensions of the design. Sets of 16 sentences, 8 involving word search and 8 requiring mixed search, were Latin-squared within this design.

Procedure. The procedure was identical to that of Experiment II, except that each subject searched for targets of all types, with target type being randomized over trials.

\section{Results and Discussion}

Mean correct RTs are presented in Table 3 for the word-search trials and mixed-search trials as a function of target type. For word search, we had expected that varying target type within-subject would remove the superiority of semantic over visual targets found in the previous experiment. Table 3 indicates that this was the case. For the mixed-search trials, our revised version of the perceptual units hypothesis predicts that a semantic-superiority effect should occur. While Table 3 indicates a trend in the predicted direction, the effect is quite small and will be shown to be insignificant.

An analysis of variance was performed on these data, and the Latin-square design again necessitated the use of Clark's (1973) min $F^{\prime}$ statistic. The only significant main effect was due to serial position, min $F^{\prime}(3,60)=7.06, p<.001$, with RTs increasing with the serial position of the critical word. Both target type and type of search (word vs mixed search) failed to produce significant effects, $\min \mathrm{F}^{\prime}<1$ in both cases. A subsequent contrast indicated that target type was still insignificant even when mixed-search trials were considered alone, $\min F^{\prime}<$ 1. Furthermore, all interactions failed to reach conventional levels of significance. Finally, the overall error rate was but $2.7 \%$, and again such a small error rate precluded a more detailed analysis.

The results of Experiment III thus provide only mixed support for our perceptual units hypotheses. On the one hand, this hypothesis correctly predicted the absence of a semantic-superiority effect in word search, but on the other, it erroneously predicted the occurrence of this effect on mixed-search trials. ${ }^{2}$

\section{GENERAL DISCUSSION}

Let us first summarize our basic proposals and findings. We began by noting Cohen's (1970) demonstration of a semantic-superiority effect, a result which seemed in conflict with the logical consideration that during reading some visual processing must precede semantic coding. Experiment I showed that this effect obtained even when the subject could not make much use of semantic context to facilitate word perception. This finding eliminatd one perceptually based explanation of the semanticsuperiority effect. Experiment I further demonstrated that the effect obtained when the visual and acoustic targets were syllables. This result excluded another perceptual hypothesis, namely, that the effect arises when visual and acoustic targets are smaller than 
syllables. However, another version of this perceptual units hypothesis, which holds that whole words are the typical processing units for words in context, was somewhat successful in predicting the results in Experiments II and III. Here, the hypothesis correctly predicted the occurrence of the semantic-superiority effect in nonword search and its absence or decrease in word search, though the hypothesis proved faulty in handling the results of mixed search.

But even if we ignore the latter problematic findings and focus only on the results for word search in Experiments II and III, the perceptual units hypothesis still leaves an important question unanswered. Why are visual targets not detected faster than semantic ones, if visual processing must precede semantic coding during reading? If our visual-target condition really tapped the initial visual coding of a word, then this condition should have been fastest of all. Having exhausted the possibilities that this failure was due to the use of inappropriately sized visual targets (letters, phonemes, syllables, and whole words have all been examined), it is time to consider the notion that visual targets in a search task are not compared to initial perceptual codes at all. That is, we wish to question the assumption that a search task like the present one can tell us anything about the perceptual units used in the initial processing of words.

The best evidence that search tasks involve units that are more abstract than perceptual codes comes from a recent series of auditory search tasks. Savin and Bever (1970) required the subject to search, i.e., to monitor, a sequence of nonsense syllables in order to detect either a target phoneme or syllable. They found that syllables were detected faster than their phoneme counterparts. Using reasoning similar to ours, they concluded that syllables were better perceptual units than were phonemes. But Foss and Swinney (1973) soon showed that such a perceptual explanation was implausible. The latter authors found that when the subject monitored a sequence of unrelated two-syllable words, two-syllable word targets were detected faster than their one-syllable counterparts, which were in turn found faster than phonemes. (This, of course, is strikingly similar to our Experiment II, where search was faster when the target matched the entire critical word than when the target comprised only one syllable of a two-syllable critical word.) Furthermore, Foss and Swinney cited data which indicated that when the subject monitors sentences, sentence targets are detected faster than word targets (a finding which has since been amply documented by McNeill and Lindig, 1973). These results led Foss and Swinney to argue that such monitoring tasks tell us nothing about perceptual units, for how can a sentence serve as a perceptual unit when there are an indefinite number of possible sentences? Rather, Foss and Swinney suggested that the results at issue were relevant to the question of which units become available to consciousness (or short-term memory, if you like) before others. The latter type of units are referred to as identification units, and are such that external responses, like the termination of a search, can be made contingent upon them. Thus, Foss and Swinney concluded that their results and those of Savin and Bever (1970) indicated that larger units of identification become available before smaller ones.

The relevance of the above proposals to the present findings is straightforward. If our search tasks involved identification units rather than perceptual ones, then there is no longer any logical reason why visual targets should be detected faster than semantic ones, as both types of targets are matched by nonperceptual units. Furthermore, the Foss and Swinney hypothesis that larger identification units become available first explains most of our principle findings. Thus, the semantic-superiority effect occurred in Experiment I and in the nonword-search trials of Experiment II because in both cases the identification units for the visual and acoustic targets were smaller than the comparable units for the semantic targets. Similarly, this effect was diminished on the word-search trials of Experiments II and III because of the increase in size of the identification units for the visual and acoustic targets. This is very similar to the explanation we had previously couched in terms of perceptual units, but the important difference is that we are now drawing conclusions about more abstract units and, consequently, it is no longer puzzling that visual targets are not detected faster than semantic ones.

Some of the puzzling aspects of other investigators' results also disappear when one assumes that search tasks reflect identification units and not perceptual ones. Consider, for example, the surprising finding that, when visually searching letter and digit arrays a subject can determine a semantic aspect of an item (i.e., whether it is a letter or a digit) before he has determined which letter or digit it is (e.g., Brand, 1971; Egeth, Jonides, \& Wall, 1972; Jonides \& Gleitman, 1972). If the determination of a specific digit or letter really reflects identification rather than perception, then the results are no longer surprising. Rather, these results indicate that a semantic aspect of an alphanumeric character may become available to consciousness early, and not that semantic information may be extracted before perception is completed (J. Balzano, personal communication, 1974).

Thus, Foss and Swinney's (1973) hypothesis seems to have fruitful implications for visual as well as auditory search. However, it should be noted that the auditory search data upon which this hypothesis is based have recently been reinterpreted by McNeill and Lindig (1973). The latter authors argue that, for example, a word target is detected faster than a 
syllable target in a search list of words because the word target is at the same linguistic level as the search list. More generally, search time decreases as the linguistic level of the target increasingly matches that of the search list, because the less the mismatch in levels, the less the subject has to divide his attention between them. Again the explanation of search processes is based on nonperceptual factors, and again it seems to be consistent with the present visual search findings. For example, consider our finding that the semantic-superiority effect occurred only in the nonword-search trials of Experiments I and II. In these cases, the linguistic level of the visual and acoustic targets was the syllable, the level of the semantic target was the word, and the level of the search list was the word or sentence. Hence, the difference in levels between semantic target and search list is less than the corresponding difference between visual or acoustic target and search list, and so the semantic target should lead to faster search times. While it is too early to tell whether this notion of matching levels will prove more valuable than Foss and Swinney's (1973) hypothesis, it seems clear that both of these nonperceptual explanations of auditory and visual search tasks merit further consideration.

To sum up, we initially investigated the semantic-superiority effect in a visual search task to see what it could tell us about the temporal relations between the perceptual and semantic codes of a word. Our results clearly indicated that this effect depended on the level of the target. While such results can be partly explained in terms of perceptual units, further considerations suggested that an explanation in terms of identification units might be more appropriate. If the latter is correct, then the import of our experiments for our understanding of the semantic-superiority effect is somewhat indirect. That is, our experiments implied that the units involved were at the level of identification, and this inference at once offers an explanation of the semantic-superiority effect and also deprives it of any mystery it might have had.

\section{REFERENCES}

Battig, W. F., \& Montague, W. E. Category norms for verbal items in 56 categories. Joumal of Experimental Psychology, 1969,80 , No. 3, Part 2.

BRAND, J. Classification without identification in visual search. Quarterly Journal of Experimental Psychology, 1971, 23, 178-186.

Clark, H. H. The language-as fixed-effect fallacy. Joumal of Verbal Learning \& Verbal Behavior, 1973, 12, 335-359.

Conen, G. A comparison of semantic acoustic and visual criteria for matching of word pairs. Perception \& Psychophysics, 1968, 4, 203-204.

Conen, G. Search times for combinations of visual, phonemic and semantic targets in reading prose. Perception \& Psychophysics, 1970, 8, 370-372.

Egeth, H., Jonides, J., \& W Al.t, S. Parallel processing of multielement displays. Cognitive Psychology, 1972, 3, 674-698.

Foss, D. J., \& Swinney, D. A. On the psychological reality of the phoneme. Journal of Verbal Learning \& Verbal Behavior, $1973,12,246-257$.

Jonides, J., \& Gleitman, H. A conceptual category effect in visual search. Perception \& Psychophysics, 1972, 12, 457-460.

Kučera, H., \& Francis, W. W. Computational analysis of present day American English. Providence, R.I: Brown University Press, 1967.

Massaro, D. W. Perceptual images, processing time, and perceptual units in auditory perception. Psychological Review, 1972, 79, 124-145.

MCNeILl, D., \& Lindig, K. The perceptual reality of phonemes, syllables, words, and sentences. Journal of Verbal Learning \& Verbal Behavior, 1973, 12, 419-430.

MorToN, J. Interaction of information in word recognition. Psychological Review, 1969, 76, 165-178.

Savin, H. B., \& Bever, T. G. The nonperceptual reality of the phoneme. Journal of Verbal Learning \& Verbal Behavior, 1970. 9. 295-302.

Smith, E. E., \& Spoehr, K. T. The perception of printed English: A theoretical perspective. In B. H. Kantowitz (Ed.), Human information processing: Tutorials in performance and cognition. Potomac, Md: Erlbaum Press, 1974.

SPOEHR, K. T., \& SMith, E. E. The role of syllables in perceptual processing. Cognitive Psychology, 1973, 5, 71-89.

Tulving, E., \& Gold, C. Stimulus information and contextual information as determinants of tachistoscopic recognition of words. Journal of Experimental Psychology, 1963, 66, 319-327.

Winer, B. J. Statistical principles in experimental design. New York: McGraw-Hill, 1971.

\section{NOTES}

1. Since Cohen's semantic task required a synonym decision in her first (1968) study and a category judgment in her context (1970) study, it is possible that the discrepancy between her two experiments may be due to synonym decisions being more difficult than category judgments. However, we know of no evidence to support this possibility. Furthermore, we will show in the following experiments that the difficulty of a category judgment, relative to the difficulties of visual and acoustic decisions, can be systematically varied without altering the category judgment itself.

2. At this point, it should be noted that our manipulations in the type of search used in Experiments II and III may be confounded with another variation. As the level of either a visual or an acoustic target is increased from nonword to word, it is possible that the similarity of the target to each comparable item in the test sentence decreases. This would mean that the comparison process used to detect a visual or acoustic target would be easier in word and mixed search than in nonword search (R. M. Shiffrin, personal communication, 1974). Such a confounding could explain why the visual and acoustic targets in the mixed-search trials of Experiment III were detected faster than expected. Furthermore, such a confounding might also account for some of the findings that we have used to support our revised perceptual units hypothesis.

(Received for publication May 24, 1974; revision received July 29,1974 .) 